\title{
Evaluando la formación virtual en sensibilización ambiental para sectores profesionales
}

\section{Assessing virtual training in environmental awareness for professional groups}

\author{
Alberto Martínez-Villar*1 \\ ecoforman@gmail.com \\ José Gutiérrez-PÉrez** \\ jguti@ugr.es \\ F. Javier Perales-Palacios** \\ fperales@ugr.es \\ *Evaluador de programas internacionales, Málaga, España \\ ** Universidad de Granada, España
}

\section{Resumen:}

En este trabajo se evalúa un programa de formación virtual (AULAVEA) centrado en la sensibilización ambiental de colectivos profesionales de sectores primarios diversos como cocina, albañilería y gestión de residuos, entre otros. En dicho programa participó una muestra internacional de estudiantes del ámbito iberoamericano seleccionados previamente. Tras una revisión de los antecedentes en esta línea de investigación se describe la plataforma diseñada al efecto. Para la evaluación de la oferta formativa se utilizó un cuestionario centrado en dimensiones propias de la evaluación de programas. El trabajo aporta una parrilla de criterios empíricos de evaluación de la calidad del modelo formativo e-learning. Los resultados evidencian un alto nivel de satisfacción con el curso implementado, consiguiendo capacitar para el desarrollo de competencias ambientales, si bien se considera que incluye una excesiva densidad informativa.

\begin{abstract}
:
In this paper a virtual training program (AULAVEA) that focused on environmental awareness of various professional groups such as cooks, builders and waste management workers, among others, was evaluated. An international sample of Latin American students was selected to participate from among a large number of applicants. Following a review of the background to this research, a platform designed for this purpose is described. To assess the training offered, a questionnaire was used concentrating on dimensions appropriate for the evaluation of training. The study provides a grid of empirical criteria in order to evaluate the quality of e-learning training model. Its results show a high level of satisfaction with the course given, which succeeded in enabling the development of environmental skills, although it was considered to include an excessive density of information. We
\end{abstract}

1 Dirección para correspondencia (correspondence address):

Alberto Martinez-Villar. C/ Retama, 32. 29190 Málaga (España) 
Se concluye planteando diversas acciones de mejora para iniciativas como esta.

Palabras clave:

Educación Ambiental; Sostenibilidad; aprendizaje mixto; TIC; educación virtual. conclude by proposing several actions to improve initiatives like this.

\section{Keywords:}

Environmental Education; Sustainability; blended learning; ICT; virtual education.

\section{Résumé:}

Cet article présente un programme de formation virtuelle (AULAVEA) axé sur la sensibilisation environnementale des groupes professionnels de divers secteurs tels que la cuisine, la maçonnerie et la gestion des déchets, parmi autres. Dans le programme ont participé des étudiants latino-américains. L'échantillon des étudiants a été dûment sélectionné Après une révision bibliographique sur ce sujet, l'article décrit le programme qui a été mis au point et utilisé dans notre recherche. Ensuite on passe á la phase d'évaluation du programme. Pour cette tâche on a utilisé un questionnaire mettant l'accent sur les dimensions spécifiques d'évaluation de programmes. Le travail fournit une grille de critères empiriques pour évaluer la qualité du modèle de formation e-learning Les résultats obtenus montrent un niveau élevé de satisfaction des étudiants envers le cours suivi. En plus, il fournit les critères empiriques pour évaluer la qualité du modèle de formation e-learning utilisé, bien qu'il soit considéré excessif à propos de la densité d'information. L'article se conclut par une série de propositions visant à améliorer les initiatives de ce genre.

Mots clés:

Éducation environnementale; Durabilité; Apprentissage mixte; TIC; Éducation virtuelle.

Fecha de recepción: 6-4-2016

Fecha de aceptación: 18-7-2016

\section{Introducción}

La situación excepcionalmente grave por la que pasa nuestro ecosistema terrestre que ha venido a denominarse "emergencia planetaria" (Vilches y Gil, 2007) obliga a multiplicar los medios para revertir ese estado de cosas. Internet constituye una herramienta de primera magnitud para la sensibilización ambiental, pero a la escasez de programas "on line" y de estudios empíricos centrados en la formación virtual de trabajadores de diferentes sectores laborales (Fauville, Lantz y Säljö, 2014; Bohlinger, Haake, Jørgensen, Toiviainen y Wallo, 2015), hay que añadir la falta de reflexiones sobre su efectividad y de indicadores de evaluación competencial (Ruiz, 2008; Martínez, 2013).

La capacitación en Sostenibilidad en el ámbito de la Formación Profesional para el Empleo (FPE) resulta un campo primordial para la cualificación de cualquier ámbito profesional en la sociedad de nuestro tiempo. A este respecto la presencia de Módulos de Sensibilización Ambiental en la FPE en España representa un importante hito que está 
logrando un incremento de la concienciación en la población que participa en estos procesos formativos. También lo es la formación continua de los trabajadores en activo y la incorporación de las empresas a la cultura de la Sostenibilidad, implementando unas Buenas Prácticas Ambientales en todas las Familias Profesionales, algo imprescindible si queremos trasladar el discurso ecologista global a acciones locales. En nuestro caso estamos interesados en evaluar estos procesos de formación con el fin de compartir recursos, intercambiar información, crear iniciativas educacionales y fomentar proyectos cooperativos.

Los altos niveles de deserción estudiantil en cursos no presenciales y el aislamiento propio de estos ambientes de formación muestran que el diálogo directo entre el docente y los alumnos constituye un factor limitante respecto a los cursos presenciales (Schneckenberg, 2004; Cabero y Llorente, 2005; Ojeda, 2009), a lo que hay que unir que la utilización de herramientas TIC no es sinónimo de calidad de enseñanza (Barberá et al., 2008). A pesar de que la tecnología constituye un medio que correctamente utilizado conduce a la consecución de los fines previstos, la mera utilización de herramientas tecnológicas avanzadas no presupone la calidad de los contenidos educativos desarrollados ni asegura de entrada aprendizajes significativos. Esta puede lograrse con una variedad de tales herramientas utilizadas de forma adecuada y apoyadas por formadores cualificados.

Algunos estudios han explorado las limitaciones del e-learning (Bartolomé, 2004; Ojeda, Gutiérrez y Perales, 2011a; Aguaded y Cabero, 2013; Ghirardini, 2014; Raposo, Martínez y Sarmiento, 2015), destacando cuatro grupos de dificultades:

1. Las que genera el modelo formativo, similares a las de la Educación a Distancia, con el añadido de los requerimientos tecnológicos que conlleva, lo que justificaría en parte el alto porcentaje de abandono de estudiantes.

2. Los defectos generados por un economicismo dominante en muchos proyectos que tratan de reducir costos en los programas virtuales, lo que se traduce en limitaciones graves como: contratación de personal con poca experiencia; asignación exagerada de número de estudiantes por tutor; uso de materiales de auto-instrucción con poco o ningún requerimiento de personal docente; énfasis en el uso de los recursos multimedia sin prestar atención a los aspectos pedagógicos, etc. 
3. La eficacia de los programas formativos depende en gran medida del interés y la motivación del alumnado, circunstancias que modulan y condicionan el aprendizaje, determinando en gran medida la consecución de resultados apropiados. Las personas aprenden cuando tienen la posibilidad de establecer relaciones con sentido de utilidad funcional entre los nuevos conocimientos y los que ya poseen, y cuando reciben la ayuda precisa para avanzar de forma autónoma e iniciar nuevos aprendizajes por sus propios medios. La interacción educativa debe apoyar esa construcción hasta que el sujeto sea capaz de buscar por sus propios medios los apoyos que necesita para construir conocimiento por sí mismo. Mantener este apoyo constante usando instrumentos tecnológicos es otro de los grandes retos del e-learning.

4. La verdadera conciencia ambiental requiere de un contacto de los estudiantes con el medio ambiente y sus problemáticas (Palmer, 1998; Palmer, Suggate, Robottom y Hart, 1999) y que dicha interacción permita el desarrollo de una alfabetización en la adquisición de competencias respetuosas "con", "sobre", "para" y "en" el medio ambiente.

Analizar algunas de estas limitaciones y posibilidades en el campo de la FPE es el objetivo de este trabajo. Ello conllevó evaluar el programa formativo que hemos desarrollado a través de la plataforma ECOFORMAN planteando una propuesta de indicadores de calidad de esta formación.

En concreto nos planteamos los siguientes objetivos generales y específicos:

Objetivos generales:

- Indagar sobre el estado de la cuestión en lo referente a la Educación Ambiental (EA) y el uso de las TIC.

- Estudiar las características propias de la enseñanza a través de ordenador y su aplicación a la EA.

- Analizar las dificultades existentes para el uso sistemático de las TIC en la actividad docente.

- Investigar las herramientas TIC disponibles para el desarrollo de programas educativos y decidir la mejor tecnología disponible. 
Objetivos específicos:

- Adquirir conocimiento de los cambios que se están produciendo en el subsistema de formación profesional dirigida a desempleados y a las personas ocupadas, en relación al uso de las TIC.

- Descifrar los elementos claves y características de las plataformas de e-learning así como para la evaluación de las acciones formativas.

- Sondear las posibilidades que ofrecen las TIC en la Formación Profesional para el Empleo y la EA.

- Adquirir referencias que muestren la importancia que hoy día está adquiriendo la Sostenibilidad como área en la que están surgiendo profesionales y cómo se está influyendo en todas las Familias Profesionales a través de la integración de la Sensibilización Ambiental.

\section{Marco teórico}

Con el impulso de la llamada sociedad del conocimiento se ha avanzado ampliamente en la integración de las TIC en la FPE. La normativa vigente en diferentes países ya no sólo contempla las acciones formativas tradicionales y presenciales, sino que de forma contundente establece la posibilidad del desarrollo de la formación mediante la modalidad "teleformación" ${ }^{2}$. Con ella se puede acceder a distintas cualificaciones profesionales de forma total o parcial, dependiendo de la cualificación, con una mínima alfabetización tecnológica y con disponibilidad de herramientas y conexión a internet. Así han ido apareciendo acciones formativas en e-learning (electronic-learning, enseñanza-aprendizaje en línea o de forma electrónica), b-learning (blended learning, modelo híbrido o mixto, donde los métodos y los recursos de la enseñanza presencial y en línea, de forma electrónica, se entremezclan) y, últimamente, m-learning (movile learning, el aprendizaje a través de dispositivos móviles o portátiles, aprender en todas partes y en todo momento sin una conexión física y permanente a redes de cable), así como otras formas de aprendizaje (Ghirardini, 2014) como el e-mentoring (asesoramiento en línea) y el e-coaching (preparación en línea).

2 En el caso de España por ejemplo la reciente Orden ESS/1897/2013 desarrolla la modalidad de teleformación para la impartición de los certificados de profesionalidad. 
Trabajos recientes sobre tendencias de investigación en el campo de la Formación Medioambiental (Ardoin, Clark y Kelsey, 2012) muestran el interés que las TIC van a tener en el futuro próximo, si bien se señala la preocupación por la desconexión de experiencias directas con la naturaleza que propician estos medios. Existe escasa literatura sobre formación para la Sostenibilidad a través de las TIC, lo que justifica nuestro interés por contribuir al impulso y mejora de este tipo de herramientas tecnológicas que, sin duda, van a ser determinantes en el compromiso ambiental y adquisición de competencias de Sostenibilidad en todas y cada una de las familias profesionales de nuestro entorno.

Algunas iniciativas internacionales que están generando cuerpos de especificaciones con el objetivo de crear contenidos digitales en formatos compatibles y normalizados, y que se plantean la reutilización de los materiales educativos y la compatibilidad entre diferentes sistemas operativos y plataformas, empiezan a ser un hecho. En el ámbito pedagógico, por el contrario, son infrecuentes las iniciativas que se han desarrollado para determinar la forma de crear contenidos digitales multimedia de calidad para su uso en la EA (Ojeda, Gutiérrez y Perales, 2011b).

No obstante, las limitaciones de acceso universal a las TIC introducen una nueva dimensión en la desigualdad que está agrandando y re-cualificando las brechas económicas, sociales y culturales ya existentes. En realidad la llamada "brecha digital" no es más que una de las injusticias producidas por la "brecha social", que es la base del problema. Sus efectos han producido lo que muchos autores de habla hispana denominan la división entre «inforricos» (aquellos que tienen acceso a un gran caudal de información) e «infopobres» (quienes permanecen marginados de ese acceso a la información). Según el informe del Programa de las Naciones Unidas para el Desarrollo 2014, el porcentaje de personas en el mundo con acceso a internet es de un 35,5\%; en los países más pobres es de un $7 \%$ y en los países más desarrollados de un $81 \%$.

Los obstáculos a vencer para que las personas puedan cambiar su entorno social y económico a través el uso de las TIC no están limitados a la existencia de una infraestructura de telecomunicación que sea accesible a un precio aceptable, aunque esta condición es por supuesto necesaria. Hay otros obstáculos como el uso del idioma propio y de la educación en general y, más específicamente, la cultura de la información. Esta cultura de la información (o cultura de redes) y la habilidad en el uso de las TIC con plena conciencia de los desafíos sociales, eco- 
nómicos, lingüísticos, culturales, políticos y ecológicos (en el sentido de la ecología de la información) no pueden adquirirse sino gracias a una formación integral y a prácticas comunitarias. Se trata de acompañar a los usuarios a ser productores de contenidos y actores de desarrollo dentro de sus comunidades, no sólo consumidores más o menos expertos (Ojeda, Gutiérrez y Perales, 2011a).

La filosofía de acceso abierto a la formación y al conocimiento ha de contribuir a desarrollar modelos de formación a la carta según las características y demandas de los diferentes sectores profesionales, a la vez que impone unos estándares de equidad y accesibilidad no sujetos a barreras de orden económico y de desigualdad social. Si bien otro tipo de barreras formativas son dignas de consideración según la tipología de usuarios.

Las actitudes de los usuarios para con los medios tecnológicos podríamos englobarlas en alguno de los perfiles siguientes, que hacen referencia al mayor o menor grado de utilización de las innovaciones tecnológicas, y que permiten caracterizar a los profesionales como: innovadores, líderes o resistentes.

Por otro lado, existe igualmente una brecha entre aquellos que prefieren que la población se eduque ambientalmente desde el mismo medio y los que admiten el uso de nuevas tecnologías, como internet; en este sentido el dilema presencial-virtual va a estar presente en los debates sobre EA de forma ineludible. La obtención de información y acceso a bases de datos con información fidedigna forma parte de las competencias de alfabetización contemporánea en temas ambientales y constituye uno de los grandes retos para los educadores ambientales, al favorecer procesos de aprendizaje basados en el contraste de información obtenida de la red sobre problemáticas globales que puede ser comparada con los problemas locales. Para los más críticos se pueden exponer una buena serie de inconvenientes morales, sociales, políticos y ecológicos en el uso de las tecnologías que requieren estudios más rigurosos (Payne, 2003).

A lo largo de los últimos años en diferentes países se han venido creando agencias, institutos e instituciones encargados de la certificación de cualificaciones profesionales que han permitido establecer catálogos de aquellas ligados a ofertas de formación reglada, continua y ocupacional, así como establecer referentes para evaluar, reconocer y acreditar los aprendizajes no formales e informales, incluida la expe- 
riencia laboral. Las normativas desarrolladas ya no sólo contemplan las acciones formativas tradicionales y presenciales, sino que de forma clara establecen la posibilidad del desarrollo de la formación mediante la modalidad denominada no presencial de "teleformación", en la que el proceso de aprendizaje de las acciones formativas se desarrolla a través de TIC telemáticas, posibilitando la interactividad de alumnos, tutores y recursos situados en distinto lugar.

En el proceso de evaluación del programa hemos considerado cuatro niveles (Kirkpatrick, 1999):

$1^{\circ}$. Reacciones de los participantes en la actividad de formación. Estas reacciones, o nivel de satisfacción, pueden referirse al contenido (relevancia, utilidad, adecuación, coherencia, credibilidad, amplitud, complejidad), al proceso (las estrategias utilizadas, la competencia del formador, los materiales utilizados, las actividades realizadas, su organización, el uso del tiempo...) o al contexto (tamaño del grupo, ambiente de aprendizaje, valores, intereses, motivaciones...).

$2^{\circ}$. Aprendizaje que se ha producido en los participantes. Se trata de conocer si la participación en la actividad de formación ha permitido que los asistentes mejoren sus conocimientos, habilidades y en algunos casos actitudes o creencias. Resulta fundamental conocer este nivel de impacto porque seguramente no podrá darse una aplicación al lugar de trabajo si no se ha producido un adecuado e interiorizado aprendizaje de conocimientos y habilidades. Las dificultades y la lentitud en el cambio de actitudes y creencias es algo que no pasa desapercibido para muchos educadores.

$3^{\circ}$. Utilización de los conocimientos, habilidades y competencias adquiridas por las personas que han participado en la formación. En este punto se ha planteado la utilidad del modelo CBAM (Concern Based Adoption Model), de diferentes etapas de preocupaciones de los profesionales (toma de conciencia, información, personal, gestión, consecuencia, colaboración y reenfoque), así como el de los diferentes momentos de niveles de uso de la innovación (no uso, orientación, preparación, uso mecánico, uso rutinario, refinamiento, integración y renovación).

$4^{\circ}$. Grado en el que la empresa o la organización mejoran su productividad, la fidelización de sus clientes, aumentan las ventas, etc. 
como consecuencia de la inversión realizada en la formación de sus empleados.

La mayoría de las evaluaciones que se realizan de los programas de formación se limita a los niveles $1^{\circ}$ y $2^{\circ}$. Aunque existe una progresiva presión por demostrar la eficacia y eficiencia de la inversión en formación, también aparecen otras fuerzas internas a las organizaciones que no favorecen una evaluación de la formación basada en el análisis de la práctica laboral. La evaluación de la formación a través de la satisfacción de los usuarios es una medida necesaria pero no suficiente para poder comprender en su extensión toda la complejidad de proceso que supone una acción formativa (Marcelo, 2011). A pesar de que la evaluación de la formación tomando como criterio el grado de satisfacción de los alumnos sea insuficiente, sí es un valor relevante para conocer cuáles son las variables que más influyen en la percepción de calidad por parte de los destinatarios.

\section{Marco empírico}

\section{La Plataforma Formativa ECOFORMAN}

La acción formativa se desarrolla en la Plataforma ECOFORMAN 3 , y supone una de las escasas experiencias disponibles actualmente en el campo de la EA y la FPE. El programa se implementa en dos fases, una de e-learning y otra de b-learning a partir de la constitución de una comunidad virtual de aprendizaje formada por profesionales en formación pertenecientes a diferentes sectores laborales, y cuenta con el apoyo de un experto en TIC y Sostenibilidad que pone a su disposición diferentes instrumentos orientados a su capacitación para la sensibilización ambiental en su sector profesional mediante el uso de un portal corporativo.

La plataforma permite: intercambiar experiencias entre expertos de diferentes sectores profesionales interesados en incorporar en su práctica laboral competencias para la Sostenibilidad y reducir los impactos ambientales, recopilar un catálogo de buenas prácticas en el campo de la formación profesional y laboral sensibilizadas con el ambiente, el uso de los recursos naturales y la minimización de emisiones y residuos, e

3 http://ecoforman.blogspot.com.es 
intercambiar experiencias inspiradoras de procesos de innovación y emprendimiento sostenible.

Del análisis realizado utilizando una ficha de catalogación y valoración de páginas web y blogs para su uso en el desarrollo de la sensibilización ambiental en la FPE, hemos creado distintos apartados en Ecoforman-Blog que permiten ir clasificando y catalogando las experiencias de forma colaborativa (figura 1).

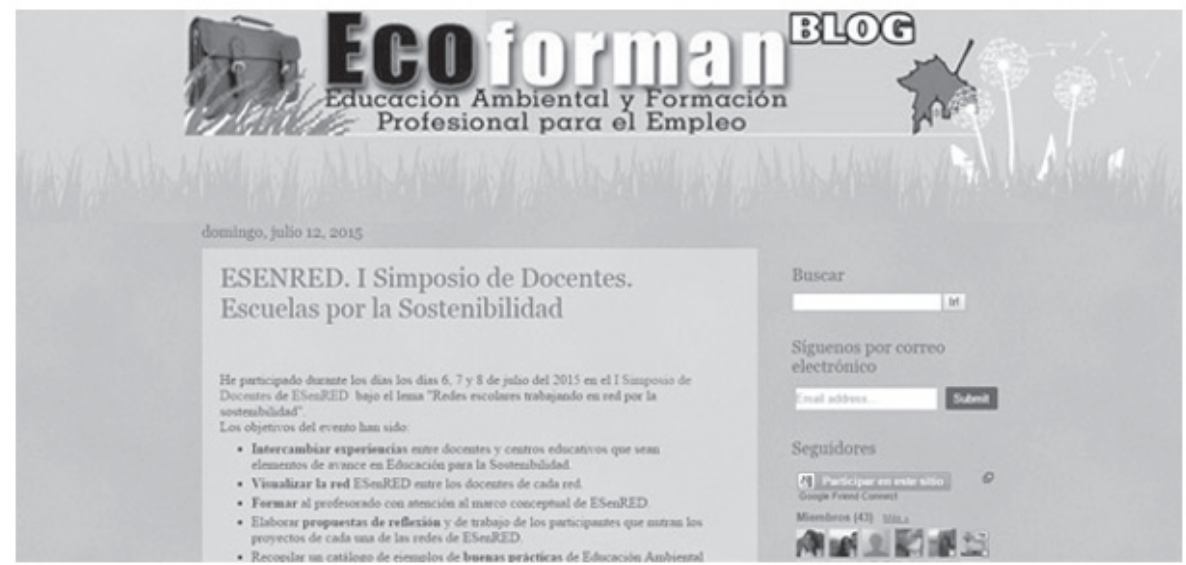

Figura 1. Página principal de Ecoforman-Blog.

Una síntesis de estos recursos nos permite agrupar la información en diferentes apartados que han sido agregados al blog en un intento de mostrar la variedad, riqueza y utilidad de los recursos, si bien somos conscientes de lo cambiante que resulta internet y de que es preciso realizar un continuo esfuerzo de actualización.

El blog se construye como un repositorio interactivo desde los presupuestos de acceso libre (open access) incluyendo recursos de software libre con el propósito de favorecer la divulgación de materiales, promover el uso de recursos sin fines comerciales sujetos a procesos de mejora interactiva continuada y hacer públicas las mejoras a los demás, de modo que toda la comunidad se beneficie de su uso.

Lo apasionante es sentirse partícipe, en igualdad de condiciones, con una comunidad de usuarios de todo el mundo dispuestos a mejorar sus competencias ambientales en cooperación con otros interesados. La plataforma ofrece un espacio dedicado a la sensibilización ambiental, a la mejora de competencias profesionales sobre Sostenibilidad y FPE. La ini- 
ciativa surge para crear un entorno de enseñanza y aprendizaje interactivo en el que personas interesadas, profesionales y expertos de distintos ámbitos y sectores laborales puedan compartir información, reflexiones, recursos, propuestas, convocatorias, etc. También pretendemos explorar y poner en marcha iniciativas que tengan que ver con las TIC al servicio de la formación en el campo de la Sostenibilidad. El sitio cuenta con más de 81.000 visitas.

En este estudio se examina una modalidad virtual de e-learning (con una fase de experimentación previa piloto de tipo b-learning). En ella intentamos indagar en las percepciones que tienen los usuarios del curso, provenientes de distintos ámbitos profesionales, sobre el proceso de enseñanza-aprendizaje llevado a cabo en el ámbito de la FPE y las TIC, mediante un cuestionario de evaluación en el que expresan su nivel de satisfacción pero también sus creencias, valores y actitudes en relación a su familia profesional a la que pertenecen. Como hemos indicado anteriormente, no existen apenas referentes de formación sobre EA a través de las TIC, con lo que queremos contribuir al impulso y mejora de este tipo de herramientas tecnológicas que sin duda van a ser trascendentes en los ámbitos profesionales ligados al Medio Ambiente, así como para la perspectiva transversal de la sensibilización ambiental en todas y cada una de las familias profesionales actualmente reconocidas.

\section{La muestra participante}

En cuanto a la muestra participante, una vez concluido el plazo de Inscripción en el curso de "Estrategias didácticas de Educación Ambiental para la Sostenibilidad a través de las TIC" se consiguieron 400 solicitudes. De todas ellas se hizo una selección preliminar de 132 y luego de las 50 definitivas. Entre los criterios de selección se tuvieron en cuenta los siguientes elementos:

- Tenía que haber "Presencia de participantes de la Comunidad Autónoma Andaluza".

- Presencia de otras comunidades españolas.

- Presencia de países iberoamericanos.

- En todos los casos se priorizan las personas que tenían experiencia y conocimientos sobre medio ambiente, sobre EA y sobre el uso de TIC.

- Se valoró la pertenencia a una organización o entidad educativa o de EA. 
Todos manifestaron su interés por la EA y su desarrollo a través de las TIC, aunque en este último aspecto algunas personas muestran más experiencia que otras. Pero todos deseosos de construir y compartir conocimiento.

De los 50 alumnos que comenzaron el curso, 25 lo han finalizado con éxito, siendo 21 los que responden a las preguntas sobre la valoración de esta acción formativa. De los 21 cuestionarios recibidos, el reparto por sexos ha sido de 11 mujeres y 10 hombres. El rango de edad de la muestra participante ha ido de 25 a 49 años, siendo el reparto por edades de 5 de 25 a 30 años, 9 de 31 a 40 y 7 de 41 a 50.

La tasa de abandono si sitúa en torno al 50\%. Estos datos no son ninguna excepción en cuanto a las tasas de abandono y éxito académico en los procesos de formación virtual.

Los países representados han sido: España, Colombia, Venezuela, México, Uruguay, Perú, Guatemala, Argentina, Ecuador, Paraguay y Puerto Rico.

\section{El cuestionario}

El objetivo es realizar una evaluación sobre la calidad de este tipo de formación basándonos en las opiniones de los usuarios, contrastándolas con estudios relevantes, y asimismo realizar una propuesta de indicadores de calidad para aquella (Ruiz, 2008; Marcelo, 2011). A su vez pretendemos enfatizar la importancia del tema elegido ya que en el momento de desarrollar la acción formativa no existía ninguna otra en ese ámbito. Somos también conscientes de la complejidad del objetivo de evaluar cuando, además, no existe un consenso generalizado sobre qué se entiende por calidad (Ehlers y Pawlowski, 2006). Como señalamos anteriormente, el uso de las TIC en contextos formativos no supone una garantía de calidad ni de mejora de los procesos de enseñanza y aprendizaje (Barberá et al., 2008:9).

La validez de contenido de la herramienta de evaluación empleada (Tabla 1) toma como base las dimensiones establecidas por estudios previos similares, aunque no ligados a las temáticas de Sostenibilidad (Ruiz, 2008:24; Marcelo, 2011:294). En nuestro caso las dimensiones en las que hemos agrupado los distintos elementos a evaluar orbitan en tres ámbitos: 
Recursos de aprendizaje: personal de apoyo, tutores, materiales para el aprendizaje, contenidos, recursos tecnológicos.

Procesos de aprendizaje: diagnóstico de necesidades, reclutamiento, diseño, desarrollo, evaluación del curso, resultados.

Contexto de aprendizaje: aspectos económicos, legales, contexto institucional, ambiente de aprendizaje, valores, motivaciones, intereses.

Tabla 1. Criterios de calidad de los instrumentos utilizados en el estudio.

\section{Criterios de Calidad}

\section{Validez /Credibilidad}

Variedad y calidad contrastada en la fuente de datos.

Consulta documental en el contexto europeo a través del Cedefop.

Consulta a expertos europeos de calidad contrastada.

Análisis directo con diversas fuentes (blog, aula virtual, comunidades de aprendizaje, grupo de trabajo, listas de correo).

Validez de contenido por expertos en la materia, miembros del equipo y por otros expertos de reconocido prestigio.

Relación de estándares para la evaluación de programas educativos.
Fiabilidad /Consistencia

Basado en metodología de estándares de calidad.

Autoridad de la base documental consultada.

Prestigio de los medios de difusión de la información.

Triangulación de referencias.

Contraste de la información.

Consolidación como informantes clave en estudios encomendados por la Junta de Andalucía.

Para la elaboración del cuestionario, hemos tenido presente una serie de consideraciones que provienen de las recomendaciones de los autores señalados. Como son:

- Realizar un encabezamiento o presentación del cuestionario para que los encuestados sepan la finalidad y el uso que se hará de los datos, así como advertir que dispondrán de los resultados de la investigación y especificar, en su caso, otro tipo de beneficios sociales que se pretenden con el estudio.

- Limitar la extensión temporal y advertir el tiempo que podrá ocupar el responder a las preguntas.

- Estructurar el modelo de respuesta para reducir al máximo lo que se tenga que escribir.

- Utilizar un estilo elocuente y términos comprensibles. Advertir que disponen de un contacto para resolver cualquier duda.

- Comprobar que el instrumento es válido y fiable. 
El cuestionario final consta de 22 preguntas de valoración del 1 al 5 (ítems y porcentajes de respuestas mayoritarias en la figura 1); y de cuatro preguntas de carácter abierto (se muestran en las figuras 2 a 5). En las preguntas de carácter abierto hemos agrupado las respuestas en torno a categorías inferidas después de haber realizado un análisis de contenido.

Los ítems de respuesta cerrada eran:

1. Fundamentación teórica

2. Tema del curso

3. Calidad de los contenidos expuestos

4. Rigor metodológico desarrollado a lo largo del curso

5. Metodología utilizada

6. Adecuación a los objetivos

7. Nivel del curso

8. Duración del curso

9. Utilidad y aplicabilidad de lo aprendido

10. Grado de participación por parte de los alumnos

11. Interacción entre los alumnos participantes

12. Calidad de las intervenciones de los tutores

13. Claridad de las explicaciones dadas por los tutores

14. Capacidad los tutores para reforzar y motivar a los participantes

15. Capacidad de los tutores para transmitir contenidos

16. Rapidez en las respuestas por parte de los monitores

17. Tareas encomendadas

18. Materiales facilitados

19. Temporalización

20. Sistema de evaluación

21. Acreditación que se da al finalizar

22. ¿Qué valoración global darías al curso?

Los ítems de carácter abierto:

1. ¿Cómo se podría evitar el abandono o falta de participación de parte de los alumnos al curso?

2. ¿Qué es lo que más te ha gustado del curso?

3. ¿Qué es lo que menos te ha gustado del curso?

4. ¿Qué podemos hacer para mejorar el curso? 


\section{Resultados y discusión}

Como puede apreciarse en la figura 2, la valoración de los elementos del programa por parte de sus usuarios es mayoritariamente positiva.

Prácticamente un $40 \%$ de las respuestas (figura 3 ) coinciden en la idea de que la mejor manera de evitar el abandono o la falta de participación en modalidades formativas como estas, reside en la interacción que se ha de establecer con los tutores y se enfatiza la necesidad de que aquellos envíen mensajes personalizados de forma continua a los participantes. Como ya hemos señalado anteriormente, el 50\% del alumnado no ha logrado finalizar el curso. Ello a pesar de que en los criterios de selección se tuvieron en cuenta factores como experiencia y conocimientos previos tanto en medio ambiente como en uso de las TIC. Hemos de considerar que este dato entra dentro de lo esperado si nos atenemos a otros estudios realizados (Cebrián, 2003; Bartolomé, 2004). En efecto, Cebrián ha señalado que el $80 \%$ de estas iniciativas suelen fracasar y que el $60 \%$ de los estudiantes abandonan los cursos. Bartolomé considera que este relativo fracaso del e-learning puede ser estudiado desde la perspectiva de las dificultades que genera el modelo subyacente y de los defectos generados por el ahorro de recursos económicos.

Teniendo en cuenta que al seleccionar la muestra potencialmente interesada, estos factores han sido controlados desde la propia fase de diseño, debemos atribuir el abandono a otro tipo de motivos, tal y como se muestra en las opiniones del alumnado y que hacen mención a aspectos motivacionales y de intereses, de planificación y de reclutamiento. Un $16 \%$ de las opiniones expresa la idea de que confluyen factores externos inevitables y que en gran medida depende del grado de motivación del alumno. También se expresa el convencimiento de que buscando fechas más idóneas se puede rebajar el nivel de abandono o de personas que no concluyen satisfactoriamente el proceso formativo. 


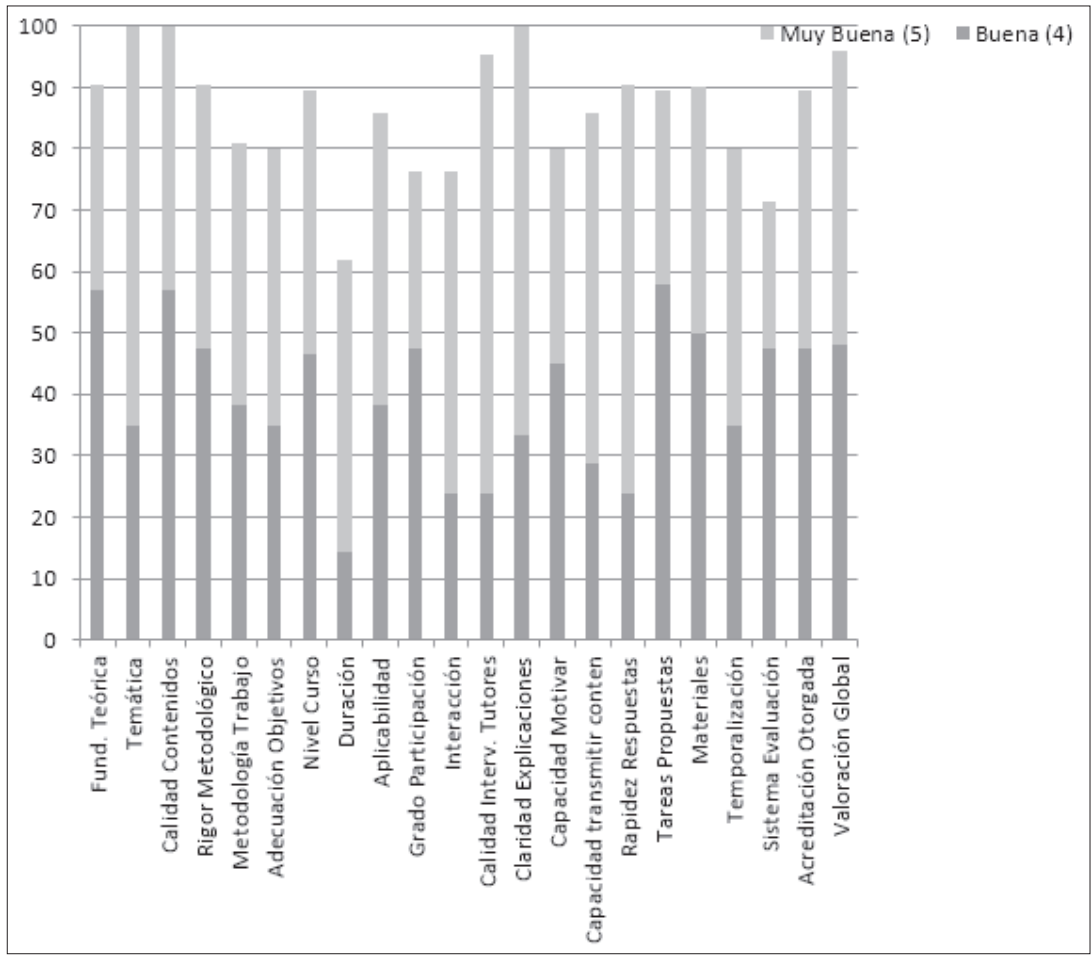

Figura 2. Resultados de la valoración del programa formativo.

En la figura 4 se pueden observar los resultados de los aspectos más exitosos del curso agrupados en categorías. Al alumnado le ha gustado sobre todo la interacción que ha existido entre los propios compañeros cuya procedencia de otros países y su calidad ha enriquecido el curso. También ha sido del agrado de los participantes en esta acción formativa la propia temática, por lo novedosa que resulta, así como la forma de presentar los distintos temas con claridad, sencillez y profundidad. En tercer lugar los participantes se muestran atraídos por los materiales generados por el profesorado y por el propio alumnado, así como con los conocimientos y comentarios de los tutores y de otros participantes expertos.

Lo que menos ha gustado del curso (figura 5) ha sido la densidad de la información en relación con el tiempo disponible. La dificultad de seguir el ritmo por parte de algunos se debe a ello, si bien en la selección de los participantes se tuvo en cuenta. El curso puede ser mejorado ampliando su duración (figura 6), dando continuidad a la acción formativa. Los participantes también expresan la idea de mejora en relación a la 
Evaluando la formación virtual en sensibilización ambiental para sectores profesionales

creación de grupos de interés facilitados por distintos tutores, creando una plataforma colaborativa y a través de un seguimiento personalizado.

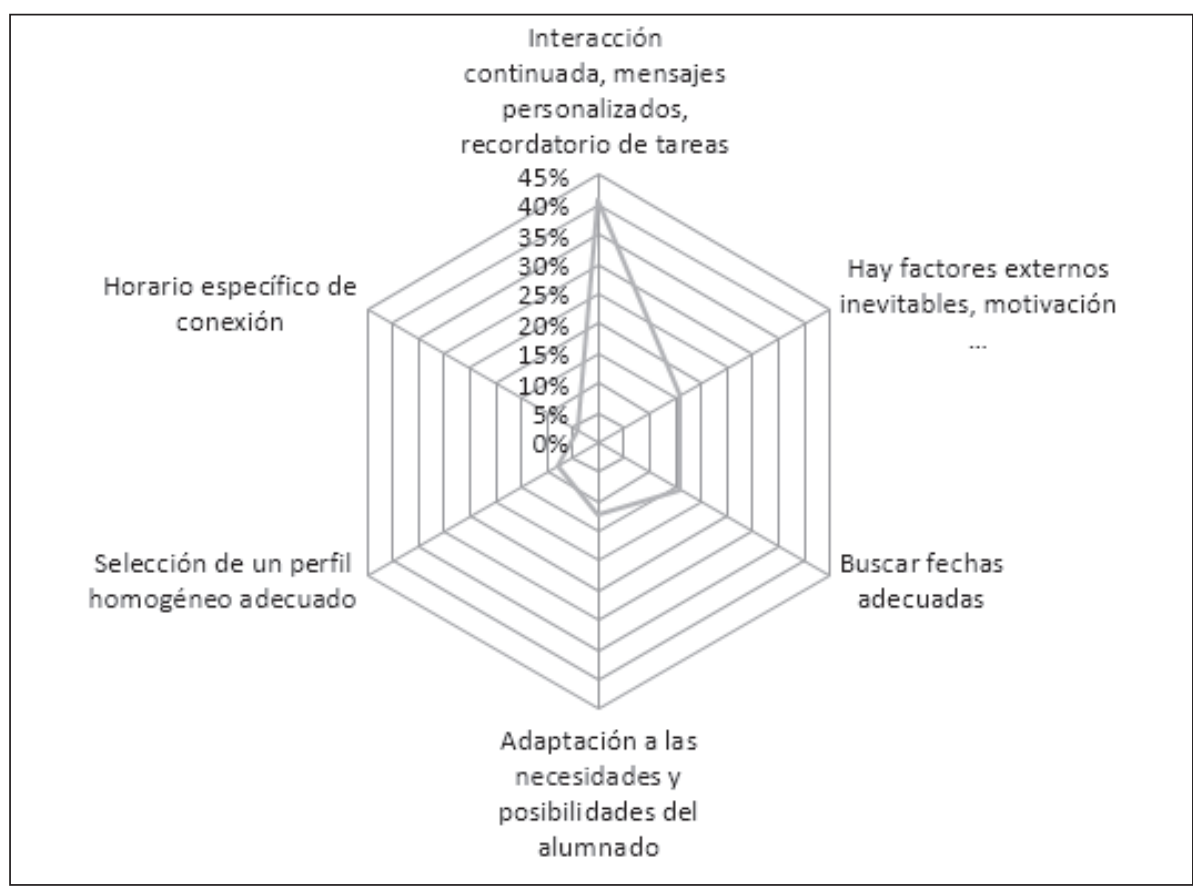

Figura 3. Cómo evitar el abandono o falta de participación de los alumnos al curso.

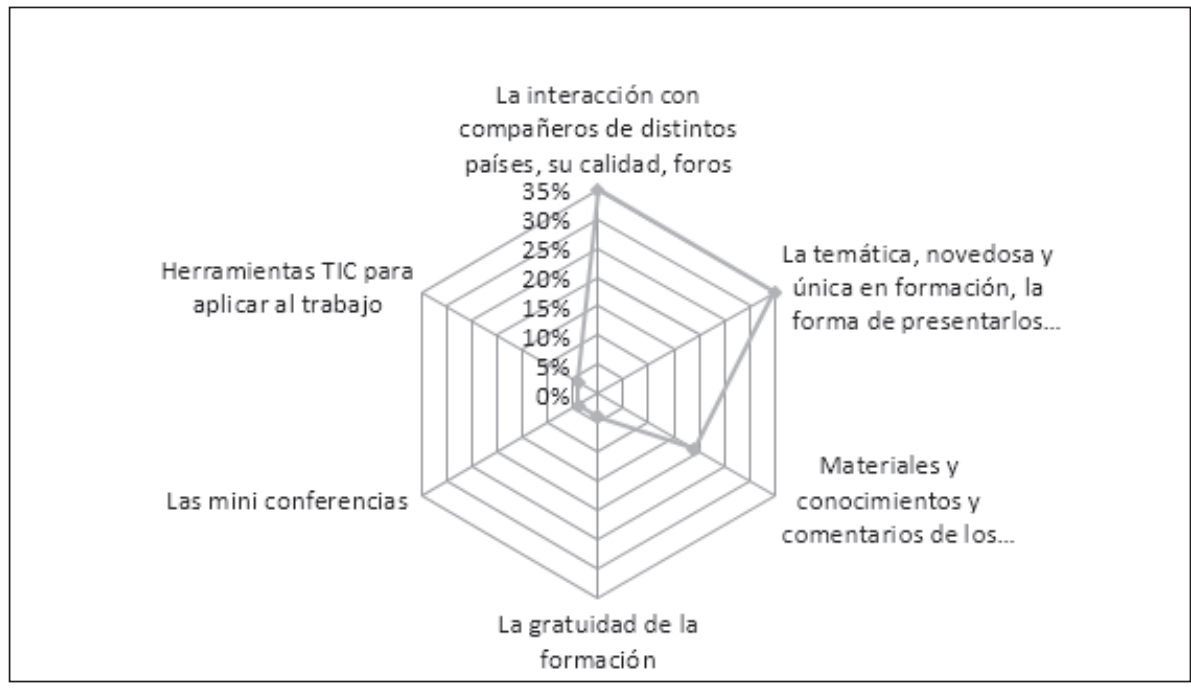

Figura 4. Aspectos más exitosos del curso. 
Evaluando la formación virtual en sensibilización ambiental para sectores profesionales Alberto Martínez-Villar, José Gutiérrez-Pérez y F. Javier Perales-Palacios

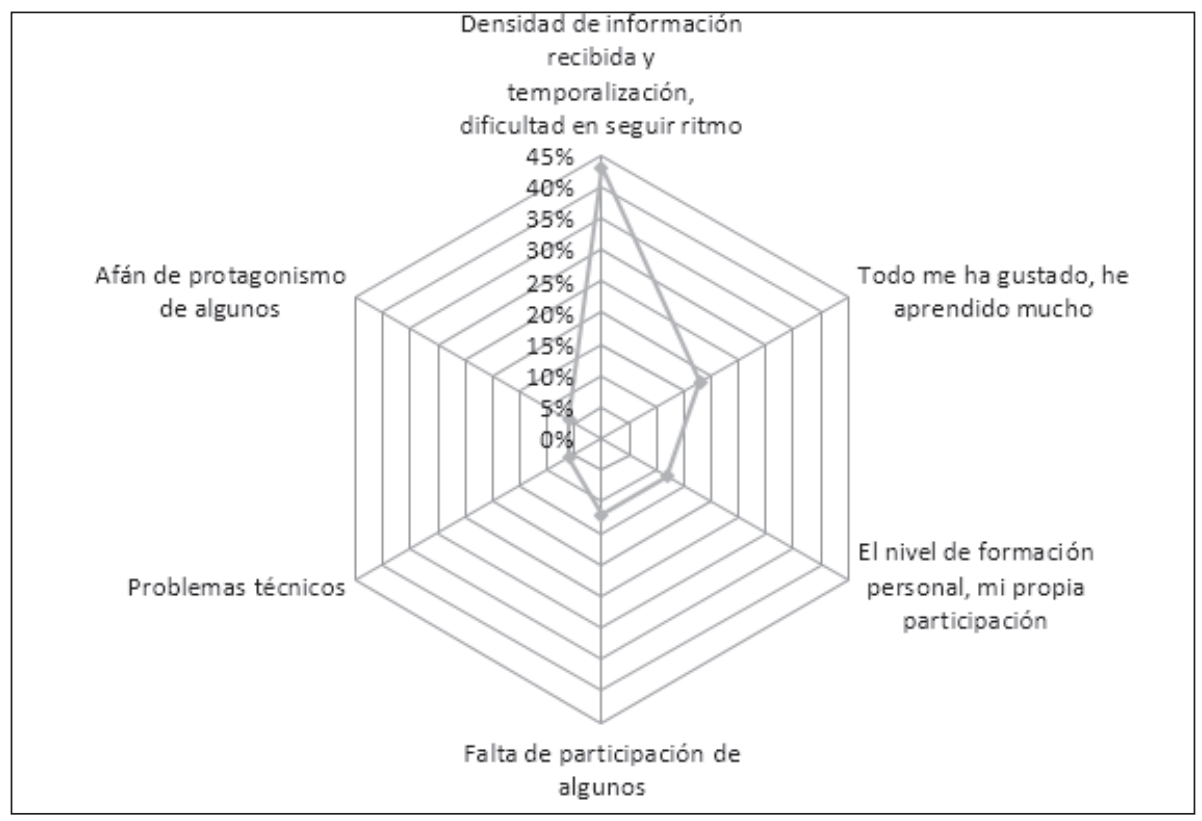

Figura 5. Aspectos menos exitosos del curso.

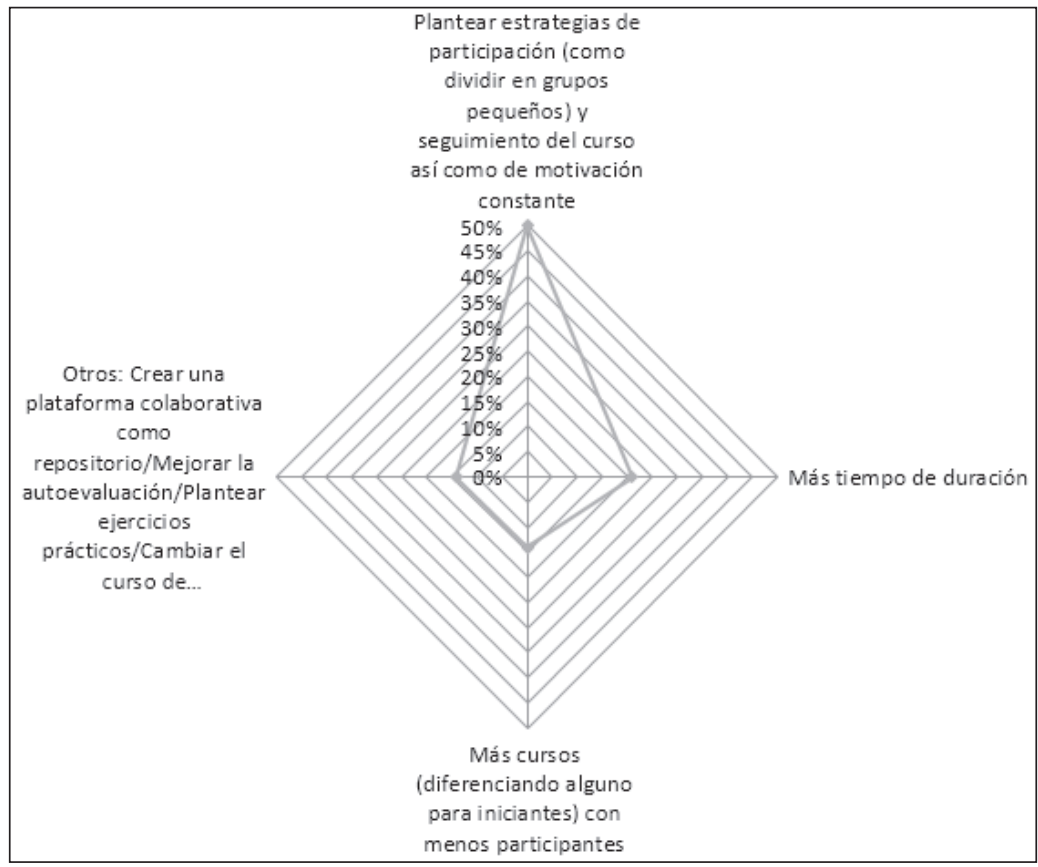

Figura 6. ¿Qué podemos hacer para mejorar el curso? 


\section{Conclusiones y recomendaciones}

La evaluación del programa formativo descrito, en cuanto a aspectos relacionados con recursos, proceso y contexto de aprendizaje, nos muestra una valoración buena o muy buena, con un valor medio global de un 8,4 sobre 10 . Somos conscientes de que los factores que prefiguran la satisfacción de los alumnos pueden ser determinantes a su vez para que el alumnado desee seguir este tipo de metodología (Sun, Tsai, Finger, Chen, y Yeh, 2008), muy útil especialmente para profesionales en ejercicio que disponen de poco tiempo para seguir formándose a lo largo de su vida laboral.

El curso podría ser mejorado proporcionándole más duración. Los participantes que han finalizado con éxito la acción formativa manifiestan expectativas de que la duración fuera mayor. Lo hemos observado en las opiniones, comentarios e intervenciones que han ido dejando en los distintos foros. En este tipo de formación el conocimiento está mediado por las TIC y el proceso de aprendizaje está centrado en el alumno y no en el profesor. El espacio para la planificación de las actividades ocupa más tiempo que el que habitualmente destinan los docentes a esta tarea. Para este tipo de enseñanza se requiere de un diseño previo bien estructurado que permita organizar el contenido, seleccionar las actividades, planificar las interacciones y esbozar la evaluación.

Entre las sugerencias para evitar el abandono o la falta de participación se menciona la interacción que se ha de establecer con los tutores y se enfatiza la necesidad de que aquellos envíen mensajes personalizados de forma continua. El hecho de ser una acción formativa única en su género, unido al hecho del interés personal que muestran los participantes que han finalizado de forma positiva, nos hace entender que aquellos que han considerado este curso como uno más, o bien han querido con ello engrosar su currículum, han sido precisamente los que no han podido o querido finalizar con éxito. Sin embargo, un $16 \%$ de las opiniones giran en torno a la idea de que hay factores externos inevitables y que en gran medida dependen del grado de motivación del alumno o incluso de las fechas en las que se realice el curso.

La elección adecuada del alumnado es también un factor determinante en cursos de esta naturaleza. Para paliar el fracaso en la formación on-line se puede adoptar un enfoque de calidad en aspectos sustantivos tales como: el enfoque del b-learning, con el objetivo de ofrecer 
una mayor flexibilidad al usuario y con el ánimo de superar las malas experiencias de los estudiantes que seguían ofertas de formación y autoformación exclusivamente virtuales (Schneckenberg, 2004:150) y el elearning, adoptando criterios de forma armónica: las necesidades y perfil académico del usuario, la naturaleza del contenido, la herramienta tecnológica y el rol mediador de un tutor on-line, guiado por principios didáctico-pedagógicos y por las teorías del aprendizaje virtual (Seoane y García, 2007).

Los participantes también expresan la idea de mejora en relación a la creación de grupos de interés facilitados por distintos tutores. Sabemos que ello supone una tarea en equipo, en la que el docente no debe estar solo, sino que necesita del apoyo de equipos multidisciplinarios, integrados por expertos en contenido, diseñadores gráficos, informáticos y pedagogos (Valeiras, 2006). Pero en muchas ocasiones nos encontramos, en acciones formativas de e-learning, con equipos que funcionan con miembros que establecen poca interacción sinérgica con otros. Se ha de dimensionar bien la densidad de la información que se ofrezca en relación al tiempo de que se disponga para trabajarla. La dificultad de seguir el ritmo por parte de algunos se debe a ello; aunque en la selección de los participantes se tuvo en cuenta, qué duda cabe que la disponibilidad de tiempo y las habilidades personales influyen en esta variable. A pesar de que en este tipo de enseñanza el conocimiento está mediado por las TIC y el proceso está centrado en el alumno y no en el profesor, en ocasiones puede que el alumnado sobrevalore sus posibilidades de uso sobre algunas de las herramientas, que sin ser difíciles de utilizar, necesitan de un tiempo de dedicación específico.

A la hora de considerar los criterios para seleccionar las TIC aplicadas a la EA coincidimos con Cabero (2006:14-15) en la importancia de considerar algunos de tipo general y en otros de tipo particular. En los generales es importante valorar que la selección de los medios debe hacerse teniendo en cuenta los objetivos y contenidos que se desean alcanzar y transmitir; las predisposiciones que el alumnado y el profesorado tengan hacia el medio pueden condicionar los resultados que se obtengan y, en consecuencia, debe de ser uno de los criterios a movilizar para su puesta en acción. También es imprescindible contemplar las características de los receptores: edad, nivel sociocultural y educativo; y las diferencias cognitivas entre los estudiantes ya que pueden igualmente condicionar los resultados a alcanzar. 
Queremos enfatizar el hecho de que la plataforma Moodle es, además, un referente que integra un marco teórico de enfoque constructivista y de aprendizaje significativo (Valeiras, 2006: 9), y que en nuestro caso ha servido para ejemplificar cómo se puede desarrollar con criterios de calidad una teleformación en distintas cualificaciones profesionales y, en particular, en una EA para la Sostenibilidad que considera una sensibilización ambiental y formación para distintas competencias (Aznar y Martínez, 2013:47) y empleos verdes. Para su implementación se ha requerido la búsqueda de financiación, un proceso de formación interna, un diseño y creación del portal, un proceso de experimentación de tipo piloto, un diseño bien estructurado que permita organizar adecuadamente el contenido, seleccionar las actividades, planificar las interacciones y diseñar la evaluación.

La utilización de los diferentes sistemas de aprendizaje propuestos estará relacionada con la disciplina a impartir y con la diversidad del alumnado. Se puede concluir la necesidad de cuatro condiciones fundamentales para que se produzca el aprendizaje a través de teleformación:

1.- Utilizar casos prácticos para hacer más significativo el contenido. El aprendizaje de los contenidos desarrollados se debe relacionar de forma muy directa con la realidad a la que se han de aplicar. Deben servir para resolver problemas que se presentan en la actividad cotidiana del trabajador. Sólo así se verán como algo más que una sucesión de ideas y conceptos y se favorecerá la motivación del alumno. El Ciclo que propone Kolb (1999) parte de la experiencia, pero a ésta le sigue la reflexión, el planteamiento de dudas y cuestiones, qué sabemos y qué desconocemos. Y sólo a partir de aquí es posible empezar a desarrollar los contenidos.

2.- Utilizar mapas conceptuales para orientar la navegación. El uso de mapas conceptuales y de otros elementos gráficos constituye un elemento fundamental en el desarrollo del aprendizaje adulto en teleformación, al estar relacionado con la perspectiva constructivista del aprendizaje. Un mapa conceptual es una representación en forma gráfica y visual de la organización y estructura de los contenidos de una acción formativa.

3.- El aprendizaje debe ser activo. En el diseño de las acciones formativas el trabajo colaborativo debe pensarse en términos de tareas. Las tareas se conciben como problemas o retos que los alumnos deben superar mediante la actividad. Esto se concreta en objetivos que podrán 
enunciarse a partir de acciones como: recordar, comprender, desarrollar, demostrar, evaluar, planificar, inventar, solucionar problemas...

4.- El cambio en los métodos de enseñanza debe ir acompañado de un cambio en la forma de evaluación. La evaluación de la acción formativa tiene como función valorar el grado de alcance de los objetivos propuestos por la misma. Es un paso del proceso formativo que merece una atención especial porque permitirá mantener y aumentar la motivación del alumno al hacerle consciente de los logros que va alcanzando, y contribuirá a mejorar constantemente los materiales formativos para subsanar las carencias de las que puedan adolecer. Las nuevas tecnologías permiten opciones de evaluación muy diversas, desde las clásicas actividades de respuesta múltiple, de respuesta única, de unir términos y similares hasta el uso de simulaciones y casos prácticos de una enorme complejidad.

De los resultados obtenidos se desprende que debieran promoverse y dinamizarse actividades relacionadas con la cualificación y el desarrollo de competencias en distintos ámbitos y sectores profesionales de la sociedad en formatos diversos (MOOCs, teleformación, e-learning, b-learning,...), intentando fomentar una deontología ambiental de las profesiones que minimice los impactos y muestre una conciencia ciudadana crítica y solidaria hacia los problemas que nos afectan. Ello debiera hacerse estimulando una participación activa que desarrolle hábitos en el aprovechamiento de los recursos naturales y la gestión de residuos, así como actitudes que promuevan una conciencia más humanizada y un disfrute del medio natural y del urbano y, por tanto, conseguir un bienestar social sin menosprecio de un progreso y desarrollo adecuados.

\section{Referencias bibliográficas}

AGUADED, J.I. y CABERO, J. (2013). Tecnologías y medios para la educación en la esociedad. Madrid: Alianza.

ARDOIN, N.M., CLARK, Ch. y KELSEY, E., (2012). An exploration of future trends in Environmental Education Research. Environmental Education Research, 19(4), 1-22. DOI: http://dx.doi.org/10.1080/13504622.2012.709823.

AZNAR, P. y MARTÍNEZ, M.P. (2013). La perspectiva de la sostenibilidad en la sociedad del conocimiento interconectado: gobernanza, educación, ética. Revista Teoría de la Educación: Educación y Cultura en la Sociedad de la Información, 14(3), 37-60.

BARBERÁ, E., COLOMINA, R., ENGEL, A., ESPASA, A., LAFUENTE, M., MAURI, T., NA- 
Evaluando la formación virtual en sensibilización ambiental para sectores profesionales Alberto Martínez-Villar, José Gutiérrez-Pérez y F. Javier Perales-Palacios

RANJO, M., ONRUBIA, J., ROCHERA, M.J., AGUADO, G., BADIA, A., COLL, C. (2008). Cómo valorar la calidad de la enseñanza basada en las TIC. Barcelona: Graó. Bartolomé, A. (2004). B-learning. Conceptos básicos. Píxel-Bit. Revista de Medios y Educación, 23, 7-20.

BOHLINGER, S., HAAKE, U., JØRGENSEN, C.H., TOIVIAINEN, H. y WALLO, A. (2015). Working and Learning in Times of Uncertainty Challenges to Adult, Professional and Vocational Education. Rotterdam: Sense Publishers.

CABERO, J. (2006). Bases pedagógicas del e-learning. Revista de Universidad y Sociedad del Conocimiento (RUSC), 3(1), 1-10.

CABERO, J. y LLORENTE, M.C. (2005). Las TIC y la Educación Ambiental. Revista Latinoamericana de Tecnología Educativa, 4(2), 9-26.

CEBRIÁN, M. (2003). Innovar con tecnologías aplicadas a la docencia universitaria, en M. CEBRIÁN (coord.), Enseñanza virtual para la innovación universitaria, pp. 21-36. Madrid: Narcea.

EHLERS, U.D. y PAWLOWSKI, J. (2006). Quality in European e-learning: An introduction, en U.D. EHELERS y J. PAWLOWSKI (eds.), Handbook on Quality and Standardisation in E-Learning, pp. 1-13. Berlin: Springer.

FAUVILLE, G., LANTZ-ANDERSSON, L.A. y SÄLJÖ, R. (2014). ICT tools in environmental education: reviewing two newcomers to schools. Environmental Education Research, 20(2),248-283.

GHIRARDINI, B. (2014). Metodologías de E-learning. Una guía para el diseño y desarrollo de cursos de aprendizaje empleando tecnologías de la información y comunicaciones. Roma: FAO.

KIRKPATRICK, D. (1999). Evaluación de acciones formativas. Los cuatro niveles. Barcelona: PISE.

KOLB, D. (1999). LSI: Learning Style Inventory, Version 9 Technical Specification. Boston, MA: Hay Group.

MARCELO, C. (2011). E-learning en la formación para el empleo: ¿qué opinan los usuarios? Revista de Educación, 355, 285-308.

MARTínEZ-VILLAR, A. (2013), La Educación Ambiental y la Formación Profesional para el Empleo. La integración de la sensibilización ambiental. Tesis Doctoral. Granada: Universidad de Granada.

OJEDA, F. (2009). Educación Ambiental y Tecnologías de la Información y la Comunicación: diseño, desarrollo y evaluación de un programa colaborativo en Educación Secundaria. Tesis Doctoral. Granada: Universidad de Granada.

OJEDA, F., GUTIÉRREZ, J. y PERALES, F.J. (2009). ¿Qué herramientas proporcionan las Tecnologías de la Información y la Comunicación a la Educación Ambiental? Revista Eureka sobre enseñanza y divulgación de las ciencias, 6(3), 318-344.

OJEDA F., GUTIÉRREZ, J. y PERALES, F.J. (2011a). TIC y Sostenibilidad: obstáculos y posibilidades para los educadores ambientales. Profesorado. Revista de Currículum y Formación del Profesorado, 15(1), 263-313

OJEDA, F., GUTIÉRREZ, J. y PERALES, F.J. (2011b). Diseño, fundamentación y validación de un programa virtual colaborativo en educación ambiental. Enseñanza de las Ciencias, 29(1), 127-146. 
Evaluando la formación virtual en sensibilización ambiental para sectores profesionales Alberto Martínez-Villar, José Gutiérrez-Pérez y F. Javier Perales-Palacios

PALMER, J.A. (1998). Environmental education in the 21st century: Theory, practice, progress and promise. Nueva York: Routledge.

PALMER, J.A. SUGGATE J., ROBOTTOM I. y HART, P. (1999). Significant life experiences and formative influences on the development of adults environmental awareness in the UK, Australia and Canada. Environmental Education Research, 5(2), 181-200.

PAYNE, P. (2003). The Technics of Environmental Education. Environmental Education Research, 9(4), 525-541.

RAPOSO, M., MARTÍNEZ, E. y SARMIENTO, J.A. (2015). Un estudio sobre los componentes pedagógicos de los cursos online masivos. Comunicar, XXXIII(44), 27-35.

RUIZ, C. (2008). El blended-learning: Evaluación de una experiencia de aprendizaje en el nivel de postgrado. Investigación y Postgrado, 23(1), 11-36.

SEOANE, A.M. y GARCÍA, F.J. (2007). Criterios de calidad en formación continua basada en e-learning. Una propuesta metodológica de tutoría on line. Actas del Virtual Campus 2006. V Encuentro de Universidades \& eLearning, pp. 37-48, Salamanca: Universidad de Salamanca.

SCHNECKENBERG, D. (2004). El e-learning transforma la Educación Superior. Educar, 33, 143-156.

SUN, P.C., TSAI, R.J., FINGER, G., CHEN, Y.Y. y YEH, D. (2008). What Drives a Successful Elearning? An Empirical Investigation of the Critical Factors Influencing Learner Satisfaction. Computer and Education, 50(4), 1183-1202.

VALEIRAS, N. (2006). Las Tecnologías de la Información y la Comunicación integradas en un modelo constructivista para la enseñanza de las ciencias. Tesis Doctoral. Burgos: Universidad de Burgos.

VILCHES, A. y GIL, D. (2007). Emergencia planetaria: necesidad de un planteamiento global. Educatio Siglo XXI, 25, 19-50. 